\title{
Location, function and role of stromal cell-derived factors and possible implications in cancer (Review)
}

\author{
WENJING GONG ${ }^{1,2}$, TRACEY A. MARTIN ${ }^{2}$, ANDREW J. SANDERS ${ }^{2}$, \\ AIHUA JIANG ${ }^{3}$, PING SUN ${ }^{1}$ and WEN G. JIANG ${ }^{2}$ \\ ${ }^{1}$ Department of Oncology, Yantai Yuhuangding Hospital, Medical College, Qingdao University, \\ Yantai, Shandong 264000, P.R. China; ${ }^{2}$ Cardiff China Medical Research Collaborative, \\ Cardiff University School of Medicine, Cardiff CF14 4XN, UK; ${ }^{3}$ Department of Anaesthesiology, \\ Yantai Yuhuangding Hospital, Medical College, Qingdao University, Yantai, Shandong 264000, P.R. China
}

Received April 30, 2020; Accepted October 29, 2020

DOI: $10.3892 / \mathrm{ijmm} .2020 .4811$

\begin{abstract}
Despite improvements in therapy and management, cancer represents and remains a major cause of mortality and morbidity worldwide. Although genetics serve an important role in tumorigenesis and tumour progression, the tumour microenvironment (TME) in solid tumours is also important and has been indicated to contribute to these processes. Stromal cell-derived factors (SDFs) represent an important family within the TME. The family includes SDF-1, SDF-2, SDF2-like 1 (SDF2L1), SDF-3, SDF-4 and SDF-5. SDF-1 has been demonstrated to act as a positive regulator in a number of types of tumour, such as oesophago-gastric, pancreatic, lung, breast, colorectal and ovarian cancer, while the biology and functions of other members of the SDF family, including SDF-2, SDF2L1, SDF-4 and SDF-5, in cancer are different, complex and controversial, and remain mainly unknown. Full identification and understanding of the SDFs across multiple types of cancer is required to elucidate their function and establish potential key targets in cancer.
\end{abstract}

\section{Contents}

1. Introduction

2. Role of SDF-1 in promoting cancer

3. Role of SDF-2 and SDF2L1 in endoplasmic reticulum (ER) stress and cancer

4. Role of SDF-4 in cancer cell mobility, proliferation and migration

Correspondence to: Professor Wen G. Jiang, Cardiff China Medical Research Collaborative, Cardiff University School of Medicine, Henry Wellcome Building, Cardiff CF14 4XN, UK E-mail: jiangw@cardiff.ac.uk

Key words: cancer, stromal cell-derived factors $1 / 2 / 4 / 5$, stromal cell-derived factor 2-like 1
5. Controversial roles of SFRP2 in the Wnt/ $\beta$-catenin signalling pathway and methylation of SFRP2 in cancer

6. Outlook and future perspectives

7. Conclusion

\section{Introduction}

The number of people diagnosed with cancer worldwide has consistently increased in recent years. There were $>18$ million new patients diagnosed with cancer and 9 million deaths caused by cancer according to 2018 GLOBOCAN (1).

Interactions between stromal, epithelial and extracellular matrix (ECM) components are increasingly recognized as being important in cancer development and progression (2). Stromal cells, together with ECM components, make up the tumour microenvironment (TME), which is vital for cancer cell proliferation, invasion and metastatic progression (2). Stromal cell-derived factors (SDFs) comprise a group of proteins derived from stromal cells (3). The family mainly includes SDF-1, SDF-2, SDF2-like 1 (SDF2L1), SDF-3, SDF-4 and SDF-5. These factors have all been identified in human cancer tissues except for SDF-3, which has been found to be present only in murine samples (3) (Table I). Although they are named similarly and belong to the same group, they exhibit different structures and functions in cancer (3). SDF-1 has been the most studied factor in this group, while less is known about the other factors and their association with cancer.

\section{Role of SDF-1 in promoting cancer}

SDF-1, a 68-amino acid protein, belongs to the chemokine family (4). Chemokines are a large family that can regulate stem or progenitor cell proliferation and movement (5). Chemokines have been classified into four main subfamilies: CXC, CC, $\mathrm{C} 3 \mathrm{XC}$ and $\mathrm{XC}$, depending on the presence and number of amino acids between $\mathrm{N}$-terminal cysteine residues (6). SDF-1 belongs to the CXC chemokine family and it is also known as CXC motif ligand 12 (CXCL12). It is expressed in various types of cancer, including oesophago-gastric, pancreatic, lung, breast, colorectal and ovarian cancer (7). As a receptor of CXCL12, CXC motif 
chemokine receptor 4 (CXCR4) has been found to be overexpressed in $>30$ types of malignant tumours (7), such as lung (4), breast (8) and intestinal cancer (9). There is increasing evidence demonstrating that CXCL12, as a secreted factor in the TME, enhances tumour growth and survival $(10,11)$, adhesion $(12,13)$, chemoresistance (4), immunotherapy resistance (14-16), migration and invasion (9,17-19), angiogenesis and metastasis (20) via the CXCL12/CXCR4 axis $(20,21)$, mainly in lung cancer, breast cancer, gastric cancer, colon cancer, pancreatic cancer, ovarian carcinoma, cervical carcinoma, endometrial cancer and leukaemia $(8,13,22-25)$. The JAK2/STAT3, MAPK, PI3K/AKT and PI3K/Pyk2 signalling pathways have been identified as acting downstream of the CXCL12/CXCR4 axis $(4,17)$. As aforementioned, the connection between CXCL12 and CXCR4 contributes to malignant behaviour. Therefore, targeting the CXCL12/CXCR4 axis may be a viable target for tumour treatment. CTCE-9908, a CXCL12 analogue, has been proven to inhibit osteosarcoma growth, adhesion and metastasis (26), as well as breast cancer cell proliferation (27). Plerixafor (AMD3100), as a CXCR4 inhibitor, competitively inhibits prostate tumorigenesis in the bone (28), decreases ovarian cancer growth and metastasis (29), prevents brain-specific metastasis (30) and protects the blood-brain barrier in patients with lung cancer (30).

It is well known that genetic polymorphisms serve a vital role in cancer pathogenesis. A meta-analysis including 17,876 participants concluded the association between SDF-1 rs1801157 polymorphism and cancer risk, demonstrating that the SDF-1 rs1801157 polymorphism may serve as a risk factor for lung and urologic cancer (31).

The function of SDF-1 has been widely investigated in cancer; however, the functions of the other stromal derived factors, SDF-2, SDF2L1, SDF-4 and SDF-5, are less known in cancer. Although they are named similarly and belong to the same family, they exhibit distinct structures and functions.

\section{Role of SDF-2 and SDF2L1 in endoplasmic reticulum (ER) stress and cancer}

SDF-2 is a small protein of 211 amino acids, consisting of protein (O-mannosyltransferase, inositol 1, 4, 5-triphosphate receptor and ryanodine receptor) domains, which are known as MIR motifs (32). Since SDF-2 lacks a hydrophobic region in addition to that at the $\mathrm{N}$ terminus, it was initially thought that SDF-2 may be a secretory, but not membranous protein (33). The SDF-2 gene is located on human chromosome 17 at qll.2, as identified using in situ hybridization, and on chromosome 11 in mice (33). SDF-2 is ubiquitously expressed in lung, breast, colon, liver and kidney $(3,33,34)$. SDF-2 in humans and mice contains a tetrapeptide at its C-terminus similar to the KDEL motif (a C-terminal sequence consisting of Lys-Asp-Glu-Leu), which characterises ER resident proteins (35). SDF2L1 is a homologue of SDF-2; it is an ER stress-inducible gene and a member of the O-mannosyltransferase protein family (35). Mouse SDF2L1 and SDF-2 sequences are 78\% similar and 68\% identical (36). SDF2L1 contains a C-terminal HDEL sequence, which is an ER retention-like motif that acts as an ER localisation signal (35). Both proteins are ER residents, but SDF-2 is constitutively expressed, whereas SDF2L1 expression is induced by ER stress (36). Since the ER is the appropriateenvironment for protein folding, secretion and quality control, ER impairment can lead to the accumulation of unfolded proteins, a phenomenon known as ER stress, activating the unfolded protein response (UPR) pathway (37). Chronic ER stress can be triggered by a number of human diseases, such as cancer, diabetes and neurodegenerative disorders (38). Similarly, an adverse cellular environment, including low nutrient levels, low $\mathrm{pH}$, oxygen deprivation and gene mutation, can also lead to ER stress (36). The UPR process acts to maintain cellular functions and sustain homeostasis, or to activate apoptosis, acting as quality control (36). The UPR process, together with other homeostatic regulatory systems, helps to minimise the disturbances in the environment (36). Schott et al (39) demonstrated that SDF2-like proteins are induced by ER-stress causing the accumulation of unfolded proteins and that they served a functional role in ER-stress, as well as in the UPR pathway. Additionally, it has been observed that following ER stress and the activation of the UPR pathway, SDF2-like proteins were increased, while silencing SDF2-like proteins led to an imbalance in the cellular environment (35). Therefore, SDF2L1 is suggested to be a critical protein in ER stress. A further understanding of the mechanisms of SDF2L1 on ER stress may be helpful in identifying its possible downstream targets in cancer (37).

There is some direct evidence that has given insight into the impact of SDF-2 and SDF2L1 in cancer. Kang et al (3) demonstrated that SDF-2 and SDF2L1 displayed differential expression patterns in patients with breast cancer. Upregulation of these factors was associated with a better clinical outcome, which is markedly different from SDF-1 (3). SDF-2 transcript levels were significantly lower in patients with metastasis $(0.0014 \pm 0.0001)$ and in those who died of the disease $(0.049 \pm 0.037)$ (3). Furthermore, using the Nottingham Prognostic Index as a prognostic factor led to the same outcome that lower transcript levels were associated with a poor prognosis (3). Similar to SDF-2, the transcript levels of SDF2L1 were also lower in patients with a poor prognosis $(0.012 \pm 0.008)$ than in patients with a good prognosis $(0.529 \pm 0.50)$ (3). Moreover, the levels of SDF2L1 in patients with metastatic disease and in those who had died from cancer were markedly lower than in patients who were disease-free, and the group with the highest tumour grade exhibited the lowest transcript levels of SDF2L1 (3). Overall, this suggested that lower levels of SDF-2 and SDF2L1 indicated a poor prognosis (3). Considering the various 'omic' technologies that have expanded the expectations for biomarkers in the cancer field, Vendrell et al (34) analysed genomics and transcriptomics data in a series of R0 Dukes B and Dukes C colorectal carcinomas to evaluate the identification of outcome predictors; the multivariate Cox model was used to identify 68 genes associated with disease-free survival. Consequently, $74 \%$ of these genes were upregulated (34). In the transcriptomic analyses, downregulation of SDF-2 expression was associated with a poor prognosis $(\mathrm{P}<0.01)(34)$. Lower SDF-2 expression was observed to be an indicator of shorter disease-free survival in colorectal cancer (34). In order to discover potential predictive biomarkers, Willis et al (40) performed a meta-analysis; the results of the meta-analysis were statistically significant with a false discovery rate $<0.05$. The results revealed that low SDF2L1 mRNA expression was associated with a poor prognosis in patients with ovarian serous carcinoma (40). 
Table I. SDFs expression in different types of tumour.

\begin{tabular}{ll}
\hline SDFs & \multicolumn{1}{c}{ Types of tumour (Refs.) } \\
\hline SDF-1 & Oesophago-gastric, pancreatic, lung, breast, colorectal and ovarian cancer (7) \\
SDF-2 & Breast cancer (3), colorectal carcinoma (34), hormone-independent tumour from a medroxyprogesterone \\
& acetate mouse breast cancer model (41) and oxaliplatin-resistant gastric cancer cells (42) \\
SDF2L1 & Breast cancer (3) and ovarian serous carcinoma (40) \\
SDF-4 & Pancreatic cancer $(48,49)$, cervical cancer HeLa cell line (44) and breast cancer (44) \\
SFRP2 & Lung cancer cells (66), choriocarcinoma (67), gastric cancer (68), prostate cancer (69), melanoma (71,79), \\
& colorectal cancer (74), liver cancer $(73)$ and breast cancer $(78)$
\end{tabular}

SDF, stromal cell-derived factor; SDF2L1, SDF2-like 1; SFRP2, secreted frizzled-related protein 2.

However, other studies have demonstrated the opposite results. For example, Giulianelli et al (41) demonstrated that SDF-2 was highly expressed in the hormone-independent tumour stroma compared with in the hormone-dependent tumour stroma from a medroxyprogesterone acetate-induced mouse breast cancer model. The aforementioned study supported the hypothesis that SDF-2 may be involved with hormone-independent tumour growth (41). Furthermore, Takahashi et al (42) revealed that SDF-2 expression was upregulated in oxaliplatin (OXA)-resistant gastric cancer cells and identified SDF-2 as a heat shock protein 72 (hsp72) client protein, which is unique to OCUM-2M/OXA cells. Hsp72 is a well-known stress-induced molecule that assists in the folding of nascent polypeptides and the refolding of denatured proteins; its constitutive overexpression enhances cancer cell stress tolerance and enables the cancer cell to adapt to harsh conditions (42). Hsp72 binds to the SDF-2 protein to promote refolding and prevents SDF-2 degradation (42). The data from the aforementioned study demonstrated that suppression of SDF-2 results in enhanced OXA-induced anti-proliferative effects and apoptosis (42). The data suggested that SDF-2 may be a novel therapeutic target in the treatment of OXA-resistant gastric cancer cells (42). Nevertheless, to the best of our knowledge there is currently no molecule that has been developed to target SDF-2 for cancer treatment. SDF-2 may potentially provide an interesting target for tumour therapies and as a prognostic factor in the future, but further studies are required.

\section{Role of SDF-4 in cancer cell mobility, proliferation and migration}

The SDF-4 protein is a $\mathrm{Ca}^{2+}$-binding protein of $45 \mathrm{kDa}(\mathrm{Cab} 45)$, and a member of the Cab45/reticulocalbin/ERC45/calumenin protein family (43). It is associated with $\mathrm{Ca}^{2+}$-dependent secretory pathways and involved in multiple diseases, including cancer (44). It consists of 361 amino acids and the corresponding gene has seven exons (45). It is located on the $1 \mathrm{p} 36.33$ chromosome (44). Cab45 has a signal sequence, six EF-hands and a HEEF motif at the C-terminal $(43,46)$. Alternative splicing produces three isoforms with different physiological and pathophysiological characters, namely the Golgi-localised variant (Cab45-G), the cytosolic variant (Cab45-C) and the secreted variant (Cab45-S) (47). Cab45-G is named according to its main localisation to the Golgi complex (44). Cab45-C is nearly identical to the 130 amino acids of Cab45-G, except that $\mathrm{Cab} 45-\mathrm{C}$ lacks the signal sequence and is localised in the cytosol (48). Cab45-C has neither a sixth-EF-hand nor a HEEF sequence, and it participates in the exocytosis of zymogen granules (48). Cab45-S is located in the ER, is secreted and differs from Cab45-G at the C-terminal sequences with no sixth EF-hand or HEEF motif (44).

Cab45 has been implicated to serve a role in numerous diseases, including cancer, where it is involved in cell migration and proliferation through a number of molecular mechanisms (44). Cab45 upregulation has been suggested in Panc1 pancreatic cancer cells (49), LIM1215 colon cancer cells (50), HeLa cervical cancer cells (44) and oesophageal cancer cells (51), suggesting that Cab45 may be involved in cancer progression. Cancer cell mobility is a vital feature in the process of invasion and migration (52). In order to promote migration and invasion, factors are secreted into the environment to provide proteins to the leading edge of a cell, which can pull the cell forward (53). The polarized orientation of the Golgi complex towards the leading edge of a migrating cell is important to ensure that factors required for persistent migratory activity are secreted at the cell's leading edge (54). It has been reported that after inhibiting vesicle formation at the trans-Golgi Network (TGN), the resultant blocking of secretion disturbs membrane delivery to the leading edge and directional cell migration (55). Cab45 has been proposed to serve a pivotal role in secretion at the Golgi complex (44). Hence, Cab45 may serve a potential role in tumour cell mobility (56).

Cab45 has also been implicated in cancer cell proliferation. A previous study revealed that Cab45 expression was significantly higher in pancreatic cancer cells compared with in non-neoplastic ductal cells using the stable isotope labelling with amino acids method (48). Additionally, Grønborg et al (49) confirmed the upregulation of Cab45 in pancreatic cancer cells, in contrast to non-neoplastic ductal cells, by immunohistochemical labelling, and indicated that Cab45 may be a potential biomarker in pancreatic tumours. Luo et al (44) arrived to a similar conclusion, revealing that there was high Cab45-G expression in the HeLa cervical cancer cell line. The aforementioned findings are in accordance with the finding that $\mathrm{Cab} 45-\mathrm{S}$ regulates the $\mathrm{Ca}^{2+}$ level of the ER by binding to sarco/ER $\mathrm{Ca}^{2+}$-ATPase $2 \mathrm{~b}$ and acts as a crucial regulator of proliferation in cervical cancer cell lines (47). It is 


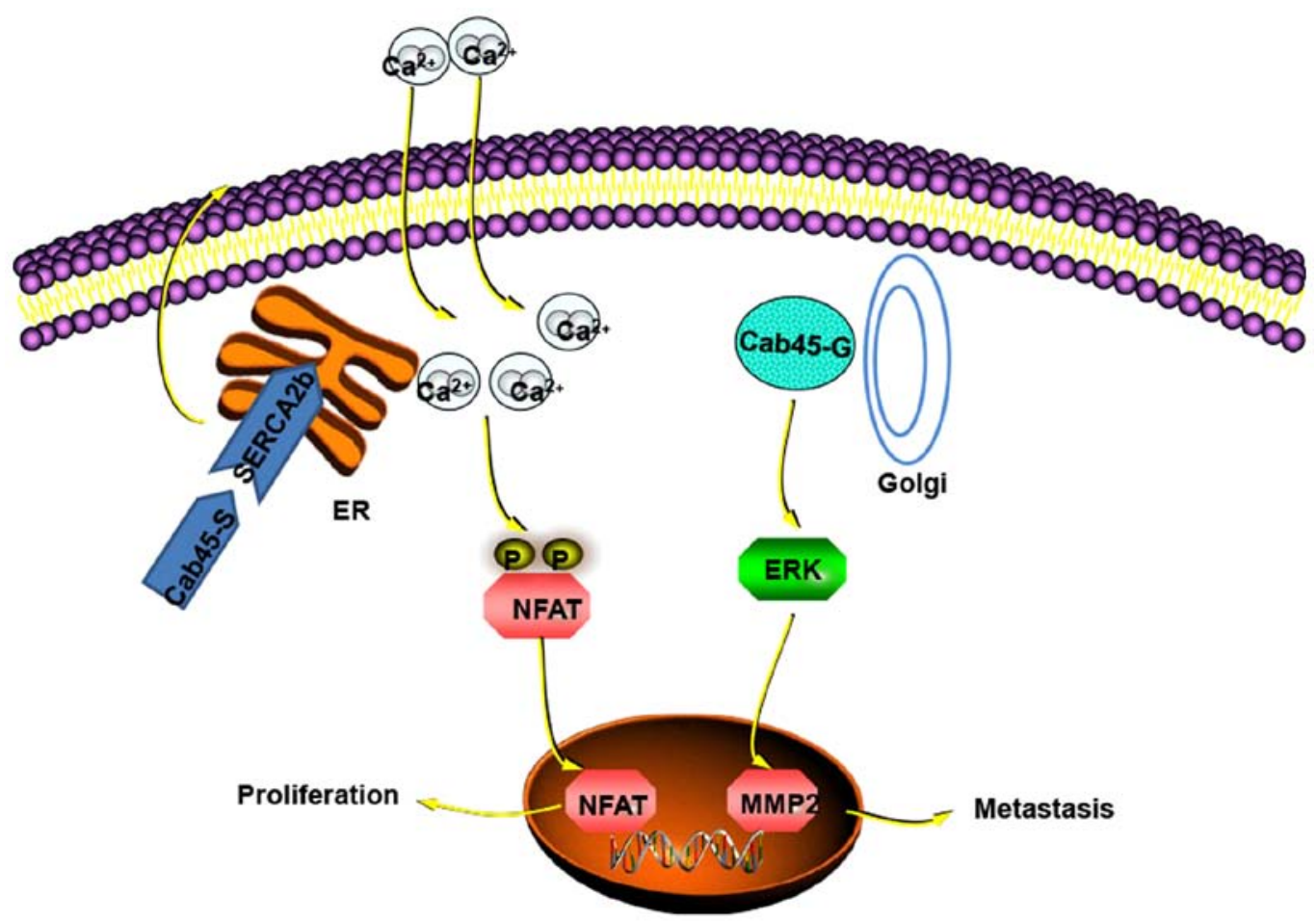

Figure 1. Function of Cab45-S in the ER and Cab45-G in the Golgi apparatus. In the ER, Cab45-S binding to SERCA2b leads to extracellular Ca ${ }^{2+}$ entry, which activates $\mathrm{Ca}^{2+}$-NFAT signalling. This causes NFAT translocation to the nucleus, resulting in cell proliferation. Cab45-G enhances cancer metastasis by increasing MMP2 expression via the ERK signalling pathway $(44,47)$. The figure was prepared utilising templates obtained from www.proteinlounge.com. ER, endoplasmic reticulum; SERCA2b, sarco/ER Ca ${ }^{2+}$-ATPase 2b; NFAT, nuclear factor of activated T cells; Cab, $\mathrm{Ca}^{2+}$-binding protein; S, secreted variant; G, Golgi-localised variant; MMP2, matrix metalloproteinase 2 .

well known that $\mathrm{Ca}^{2+}$ serves a vital role in cellular proliferation. Cab45-S can lead to nuclear translocation of the nuclear factor of activated T cells (NFAT) by increasing $\mathrm{Ca}^{2+}$ levels that result in cell proliferation (47) (Fig. 1). Intracellular levels of $\mathrm{Ca}^{2+}$ are limited, with prolonged bouts of signalling dependent on the influx of external $\mathrm{Ca}^{2+}$ via the store operated $\mathrm{Ca}^{2+}$ channels (SOCs) in the plasma membrane (50). Weiss et al (57) demonstrated that the anti-proliferative effect of non-steroidal anti-inflammatory drugs (NSAIDs) in colorectal cancer (CRC) cells was enhanced when the entry of SOCs was inhibited. Additionally, Ji et al (50) revealed that, using difference gel electrophoresis analysis, when the CRC LIM1215 cell line was treated with sulindac, a type of NSAID, Cab45 was secreted. A similar conclusion was confirmed by western blot analysis in other CRC cell lines, such as HCT116, SW480 and SW1463 (50). Therefore, it may be hypothesised that Cab45 may participate in SOCs entry to regulate the effect of NSAIDs on CRC cells and that Cab45 upregulation may be associated with tumour cell proliferation.

Finally, Cab45-G was found to promote cell migration and metastasis. The process of tumour migration and metastasis is complex and involves numerous biological aspects, including the effect of epithelial-mesenchymal transition (EMT), matrix metalloproteinases (MMPs) and ECM proteins. EMT is a critical process in epithelial cells, allowing them to attain migratory and diffusive abilities (58). During EMT, epithelial cells lose cell-to-cell junctions and apico-basal polarity, and develop an enhanced migratory capacity and upregulate $\mathrm{N}$-Cadherin and $\beta$-catenin expression, which then leads to tumour cell migration (53). Firstly, Cab45 contributes to the enhanced expression levels of EMT-associated proteins, including N-Cadherin, $\beta$-catenin and vimentin, in breast cancer, while the levels of E-Cadherin are decreased (44). In addition, Cab45-G has been associated with MMPs in human cervical cancer tissues (44). MMPs contribute to the cleavage of the matrisome (global composition of the ECM proteome) and of proteins that are responsible for ECM remodelling, causing ECM degradation (59). Additionally, MMPs activate some bioactive molecules, including cytokines, growth factors and receptors, which have been proved to promote tumour progression and metastasis (60). Cab45-G was demonstrated to be significantly associated with MMP2 expression, which has been implicated in tumour metastasis by activating the ERK signalling pathway in HeLa cells (44) (Fig. 1). Similar findings have revealed that Cab45 is responsible for successful sorting of a subset of proteins at the TGN for cell migration, such as the ECM-associated proteins matrix Gla protein, thrombospondins 1 and 3, and MMP2 (56). Therefore, the aforementioned studies suggest that Cab45-G may regulate cell migration through ECM-associated proteins, MMPs and molecular mediators of EMT $(44,56,58,60)$.

Overall, Cab45 may serve as a therapeutic target for cancer treatment and a potential predictor. However, to the best of our knowledge, no studies have been performed to explore the corresponding targeted therapy to Cab45.

\section{Controversial roles of SFRP2 in the Wnt/ $\mathbf{\beta}$-catenin signalling pathway and methylation of SFRP2 in cancer}

SDF-5 was initially discovered from a cDNA library from a murine bone marrow stromal cell line (61). Kang et al (3) 


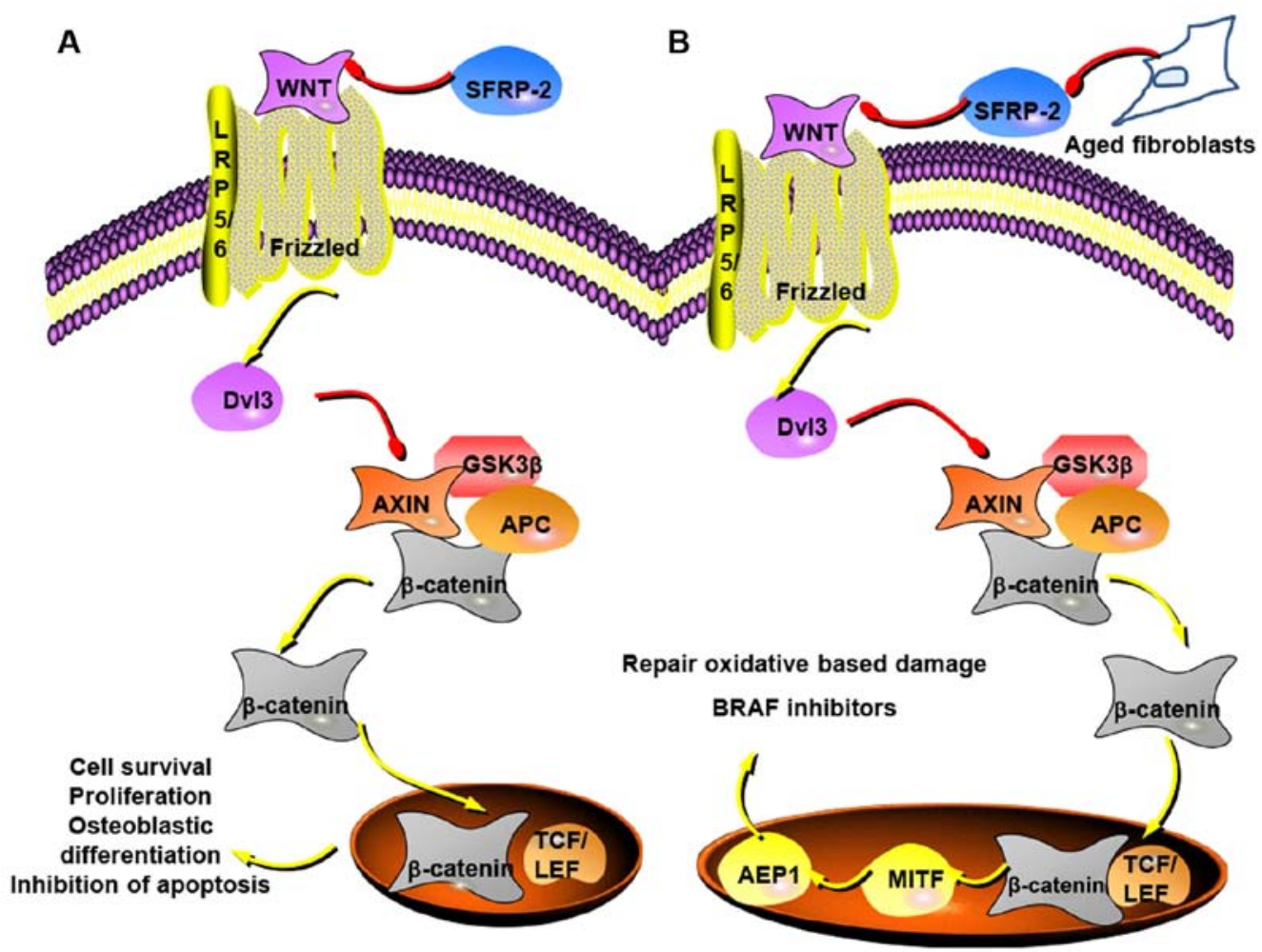

Figure 2. SFRP-2 in the Wnt/ $\beta$-catenin signalling pathway in cancer. (A) Wnt binding to Frizzled/LRP5/6 induces Dvl3 accumulation, which inhibits the complex of $\beta$-catenin, Axin, APC and GSK-3 $\beta$. This leads to an increase of cytoplasmic $\beta$-catenin, which then translocates to the nucleus to interact with TCF/LEF family of transcription factors that contribute to cell survival, proliferation and osteoblastic differentiation. SFRP-2 as a competitive receptor of Wnt may regulate the Wnt/ $\beta$-catenin signalling pathway to inhibit cell proliferation and survival, and lead to apoptosis (63,64). (B) SFRP-2 derived from aged fibroblasts may inhibit $\beta$-catenin and MITF signalling, resulting in a decrease of AEP1 that makes melanoma cells more sensitive to oxidative stress and resistant to BRAF inhibitors (74). The figure was prepared utilising templates obtained from www.proteinlounge.com. SFRP-2, secreted frizzled-related protein 2; LRP5/6, lipoprotein receptor-related protein 5/6; Dvl3, dishevelled 3; APC, adenomatosis polyposis coli; GSK-3 $\beta$, glycogen synthase kinase-3 $\beta$; TCF/LEF, T-cell factor/lymphoid enhancer factor; MITF, microphthalmia-associated transcription factor; AEP1, apurinic/apyrimidinic endonuclease 1.

reported that SDF-5 transcripts were decreased in breast cancer, but little knowledge is available with regard to SDF-5 function. SDF-5 belongs to the secreted frizzled proteins and it is similar to secreted frizzled-related protein 2 (SFRP2) (36). SFRP2 was identified as a member of the SFRP family (62). SDF-5 is homologous to Frizzled, which is the extracellular portion of the Wnt receptor (62), and so is able to compete with Frizzled receptors in interactions with Wnt proteins (63).

$\mathrm{Wnt} / \beta$-catenin signalling serves a key role in various biological processes including carcinogenesis, embryonic development and neurodegenerative diseases (62). It is highly recognised that $\mathrm{Wnt} / \beta$-catenin signalling is activated in various types of human cancer, including non-small cell lung cancer (NSCLC), melanoma and colorectal cancer. When the Wnt ligand binds to the Frizzled/lipoprotein receptor-related protein $5 / 6$, the $\mathrm{Wnt} / \beta$-catenin signalling pathway is activated and dishevelled 3 simultaneously starts to accumulate, which inhibits the combination of $\beta$-catenin, Axin, adenomatosis polyposis coli and glycogen synthase kinase- $3 \beta$; this in turn suppresses phosphorylation and degradation of $\beta$-catenin (63). Consequently, the increased accumulation of $\beta$-catenin in the cytoplasm results in its translocation to the nucleus, where it interacts with members of the T-cell factor/lymphoid enhancer factor family of transcription factors to stimulate the expression of genes involved in cell survival, proliferation and osteoblastic differentiation (63,64) (Fig. 2A).
Increasing evidence has demonstrated that SFRP2 can regulate $\mathrm{Wnt} / \beta$-catenin signalling in tumours, acting as an antagonist and contributing to the inhibition of tumour malignancy (65). SFRP2 expression is downregulated in the NSCLC A549 cell line compared with in a normal pulmonary epithelial cell line (65). Furthermore, SFRP2 may inhibit the survival and metastasis of NSCLC cells via the Wnt//-catenin signalling pathway (66). Zeng et al (67) reported that SFRP2 expression was downregulated in choriocarcinoma, and low SFRP2 expression induced tumour migration and invasion via Wnt/ $\beta$-catenin signalling. Cheng et al observed the same phenomena in gastric cancer, in which SFRP2 expression was decreased in tumour tissues compared with in adjacent non-cancer tissues (68). Additionally, Perry et al (69) revealed that SFRP2 expression was lower in prostate cancer tissues compared with in normal tissues $(\mathrm{P}<0.01)$. Moreover, it has been demonstrated that increasing the expression levels of SFRP2 can inhibit cancer cell proliferation and lead to cell apoptosis in vivo (68).

The methylation of SFRP2 serves an important role in tumour invasion and metastasis. Methylation of gene promoter regions, mainly located in $\mathrm{CpG}$ islands, is a common feature in human cancer, where it typically leads to epigenetic silencing of tumour suppressor genes (70). SFRP2 is often methylated in several types of human cancer, such as melanoma, gastric carcinoma, colorectal, liver and lung cancer (63,71-73). In 
an analysis of SFRP2, which included 10 studies, 6 studies compared 936 patients with CRC and 794 normal colonic mucosa, and 763 patients with CRC and 487 benign mucosal lesions. The data demonstrated that SFRP2 methylation in $\mathrm{CRC}$ was higher than in normal colonic mucosa and benign mucosal lesions [odds ratio $(\mathrm{OR})=31.38$ and $\mathrm{P}<0.001$, and $\mathrm{OR}=4.83$ and $\mathrm{P}<0.001$, respectively] (74), indicating that SFRP2 methylation may be used as a non-invasive biomarker for the diagnosis of CRC. Zhang et al (65) indicated that, compared with in normal lung tissues, SFRP2 mRNA expression was significantly decreased in NSCLC tissues, while the methylation of the SFRP2 gene displayed the opposite trend. In NSCLC cell lines, the demethylation of the SFRP2 gene aided in the restoration of SFRP2 expression at the RNA and protein levels (65). Therefore, tumour cell invasion may be inhibited by the stimulation of SFRP2 (65). It is reported that the demethylation of the SFRP2 gene appeared to inhibit two key factors, zinc finger E-box binding homeobox 1 (ZEB1) and MMP9 (65). ZEB1 is a promoter involved in EMT, while MMP9 is an important MMP family member that leads to the degradation of the basement membrane, which results in tumour metastasis (65). SFRP2 may therefore suppress NSCLC invasion by inhibiting ZEB1 and MMP9, and SFRP2 methylation may promote NSCLC invasion (65).

Numerous studies have indicated that SFRP2 acts as an anti-oncogene $(65,66,68-70)$; however, other studies have suggested the opposite and that it serves as an oncogene to promote carcinoma development and progression (63,75-77). In breast cancer, SFRP2 expression is upregulated in canine mammary gland tumours compared with in normal tissues (78). Lee et al (77) indicated that SFRP2 inhibited canine mammary gland tumour UV- induced apoptosis via activation of the NF- $\kappa B$ signalling pathway or by suppressing the JNK signalling pathway. Additionally, SFRP2 serves a role in leading cell adhesion and anti-apoptotic functions by integrating with the fibronectin-integrin protein complex in mammary tumours (75). Furthermore, SFRP2 was proposed to lead to tumour growth in glioma and renal cancer via activation of canonical Wnt signalling, or to promote angiogenesis through calcineurin/NFAT in breast cancer (76). Data suggests that as dermal fibroblasts grow old, SFRP2 expression increases as an antagonist of the Wnt signalling pathway to drive melanoma cell metastasis (79). SFRP2 inhibits $\beta$-catenin and microphthalmia-associated transcription factor signalling, which is a main regulator of melanoma metabolism; this in turn decreases the redox regulator apurinic/apyrimidinic endonuclease 1, which is involved in repairing oxidative-based damage, makes melanoma cells more sensitive to oxidative stress and drives resistance to BRAF inhibitors, which are an effective treatment of metastatic melanoma (79) (Fig. 2B). In elderly patients, the TME is more likely to activate signalling pathways that drive more aggressive melanoma cell behaviour (79). It has previously been reported that downregulating SFRP2 results in a decrease of breast cancer tumour volume to $46 \%$ compared with control tissues in mice (80). A similar trend was observed in lung cancer, where overexpression of SFRP2 in the NSCLC A549 cell line promoted lung cancer cell proliferation (81). After downregulating SFRP2, both CDK4 and cyclin D1 mRNA and protein expression was downregulated, and cell proliferation was suppressed at the $\mathrm{G}_{1}$ phase (81). Considering that when cyclin D1 binds to its activator $\mathrm{CDK} 4$, it enables the cell cycle $\mathrm{G}_{1}$ checkpoint to continue, previous data suggests that SFRP2 may serve a role in enhancing lung cancer cell proliferation, invasion and migration (81). Additionally, Liu et al (63) revealed that overexpression of SFRP2 in lung cancer may promote tumour diffusion and availability of Wnt proteins, resulting in activation of canonical Wnt signalling and tumorigenesis.

Accumulating studies have demonstrated that SFRP2 regulation is associated with Wnt signalling activity and tumour progression, including lung cancer, choriocarcinoma, gastric cancer, prostate cancer, colorectal cancer and melanoma (66-69,71,74). SFRP2 has the potential to act as a methylation biomarker for the diagnosis of NSCLC (72). It functions as an oncogene or anti-oncogene in numerous types of cancer, and whether it acts as an antagonist or agonist in Wnt signalling remains controversial. Regarding the therapeutic aspects, due to the inhibitory functions of SFRP2 observed in the majority of studies (65,66,68-70), SFRP2-like molecules or drug inhibitors should be explored to target tumour cells and inhibit Wnt signalling. XAV939, a Wnt inhibitor, has been demonstrated to reverse the EMT phenotype and stemness markers, resulting in the inhibition of migratory and invasive abilities in choriocarcinoma (67). Moreover, due to the role of SFRP2 methylation in promoting tumour progression, drugs that reverse or prevent this methylation may be helpful for tumour therapy. For example, 5-Aza-2'-deoxycytidine is able to cause promoter demethylation of SFRP2 and has been demonstrated to inhibit tumour migration and invasion in choriocarcinoma (67). Similarly, in addition to agents that inhibit Wnt signalling and activation of the SFRP2 gene, therapies that activate silenced genes, such as SFRP2, epigenetically may be of interest (82). DNA methyltransferases (DNMTs) contribute to the epigenetic regulation, and novel drugs targeting DNMTs are the subjects of scientific research in ongoing clinical trials in different types of cancer (63).

\section{Outlook and future perspectives}

For several decades, it was considered that the process of tumour development was associated with genetic alterations. There is now growing recognition that the TME serves an essential role in the process of cancer development. SDFs, which are derived from stromal cells, have an impact in tumour-stroma networks in cancer. SDF-1 has been the subject of most scientific research and is involved in the progression of various types of cancer and stages, including tumorigenesis, metastasis and survival. SDF-2 and SDF2L1 are ER-resident proteins associated with ER stress. SDF2L1 expression is upregulated by ER stress and SDF-2 is constitutively expressed. They may function as negative regulators in cancer through activation of the ER stress to balance the cellular environment. It has been demonstrated that cancer cells may overcome this mechanism, preventing ER stress-induced apoptosis (83). Additionally, it has been demonstrated that SDF-2 can promote acquisition of OXA resistance via enhancing the ER stress to avoid OXA-induced stress (42). These results make it difficult to understand the role of SDF-2/SDF2L1 in cancer and therefore future work is required to identify and fully establish the function of SDF-2/SDF2L1 in tumour development, ER stress and drug resistance. This elucidation may lead to potential therapeutic targets for novel cancer treatments. However, no drug or SDF-2-like therapeutics have currently been developed 
to target SDF-2 for cancer treatment. The splicing isoforms of SDF-4 (Cab45) may regulate cancer cell mobility, proliferation and migration through various mechanisms, including by regulating EMT, MMPs and ECM, but the underlying molecular mechanisms surrounding this regulation remain unclear. SDF-4 appears to have promise as a therapeutic target for the treatment of cancer, but further studies are required to fully explore the associated mechanisms. Based on the majority of previous studies $(65,66,68-70)$, SFRP2 functions as a negative regulator in tumour growth and metastasis in a number of types of cancer; however, there are contrasting results. Overall, the specific role of SFRP2 in cancer remains unclear. The function of SFRP2 may be associated with different signalling pathways, different stages of tumour progression, human age and different types of tumour. Defining the exact function of SFRP2 in different types of cancers requires to be further elucidated. If this can be clarified, SFRP2 may serve as a potential target for cancer therapy. A number of studies have demonstrated that SFRP 2 serves a pivotal role in the Wnt signalling pathway and cancer $(65-67,76,79)$. SFRP2-like therapeutics or drug inhibitors warrant scientific attention for their capacity to target tumour cells and inhibit Wnt signalling. Although there is currently no Wnt inhibitor used in the clinical setting, there have been increasing therapeutic approaches under research, such as promoters of SFRP2 activation, Wnt inhibitors and DNA methyltransferases. Further studies regarding the role of SFRP2 in cancer are required to highlight its potential for tumour treatment.

\section{Conclusion}

Overall, SDFs serve an important role in tumorigenesis and tumour progression. SDF-1 is widely known to enhance tumour malignant behaviour. SDF-1 analogues or CXCR4 inhibitors have been demonstrated to inhibit tumour growth and metastasis. SDF-2 has some potential as a predictive biomarker in tumours, but little progress has been made on the development of treatments targeting SDF-2. SDF-4 (Cab45) has been observed to enhance tumour cell mobility, proliferation and metastasis, and may therefore serve as a potential target for tumour treatment. However, further studies are required to explore the therapeutic potential of targeting Cab45. SFRP2 has been observed to serve a controversial role in cancer. The majority of previous studies suggest that it functions as an antagonist, and there have been some molecular therapeutic interventions targeting SFRP2 or Wnt signalling under research, which may provide new tumour treatments in the future.

\section{Acknowledgements}

Not applicable.

\section{Funding}

This study was supported by the Cardiff University China Medical Scholarship and the Outstanding Young Scholarship from Yantai Yuhuangding Hospital (grant no. YDH050719).

\section{Availability of data and materials}

Not applicable.

\section{Authors' contributions}

WG wrote the manuscript. TAM, AJS and WGJ contributed to manuscript preparation. PS, WGJ and AJ were involved in the conception of the study. All authors read and approved the final manuscript.

\section{Ethics approval and consent to participate}

Not applicable.

\section{Patient consent for publication}

Not applicable.

\section{Competing interests}

The authors declare that they have no competing interests.

\section{References}

1. Bray F, Ferlay J, Soerjomataram I, Siegel RL, Torre LA and Jemal A: Global cancer statistics 2018: GLOBOCAN estimates of incidence and mortality worldwide for 36 cancers in 185 countries. CA Cancer J Clin 68: 394-424, 2018.

2. Micke $\mathrm{P}$ and Ostman A: Tumour-stroma interaction: Cancer-associated fibroblasts as novel targets in anti-cancer therapy? Lung Cancer 45 (Suppl 2): S163-S175, 2004.

3. Kang H, Escudero-Esparza A, Douglas-Jones A, Mansel RE and Jiang WG: Transcript analyses of stromal cell derived factors (SDFs): SDF-2, SDF-4 and SDF-5 reveal a different pattern of expression and prognostic association in human breast cancer. Int J Oncol 35: 205-211, 2009.

4. Wang M, Lin T, Wang Y, Gao S, Yang Z, Hong X and Chen G: CXCL12 suppresses cisplatin-induced apoptosis through activation of JAK2/STAT3 signaling in human non-small-cell lung cancer cells. Onco Targets Ther 10: 3215-3224, 2017.

5. Miller MC and Mayo KH: Chemokines from a structural perspective. Int J Mol Sci 18: 2088, 2017.

6. Mélik-Parsadaniantz S and Rostène W: Chemokines and neuromodulation. J Neuroimmunol 198: 62-68, 2008.

7. Samarendra H, Jones K, Petrinic T, Silva MA, Reddy S, Soonawalla Z and Gordon-Weeks A: A meta-analysis of CXCL12 expression for cancer prognosis. Br J Cancer 117: 124-135, 2017.

8. Ling X, Spaeth E, Chen Y, Shi Y, Zhang W, Schober W, Hail N Jr, Konopleva M and Andreeff M: The CXCR4 antagonist AMD3465 regulates oncogenic signaling and invasiveness in vitro and prevents breast cancer growth and metastasis in vivo. PLoS One 8: e58426, 2013.

9. Kollmar O, Rupertus K, Scheuer C, Junker B, Tilton B, Schilling MK and Menger MD: Stromal cell-derived factor-1 promotes cell migration and tumor growth of colorectal metastasis. Neoplasia 9: 862-870, 2007.

10. Kryczek I, Wei S, Keller E, Liu R and Zou W: Stroma-derived factor (SDF-1/CXCL12) and human tumor pathogenesis. Am J Physiol Cell Physiol 292: C987-C995, 2007.

11. Wu M, Chen Q,Li D,Li X,Li X, Huang C, Tang Y,Zhou Y, Wang D, Tang K, et al: LRRC4 inhibits human glioblastoma cells proliferation, invasion, and proMMP-2 activation by reducing SDF-1 alpha/CXCR4-mediated ERK1/2 and Akt signaling pathways. J Cell Biochem 103: 245-255, 2008.

12. Dehghani M, Kianpour S, Zangeneh A and Mostafavi-Pour Z: CXCL12 modulates prostate cancer cell adhesion by altering the levels or activities of $\beta 1$-containing integrins. Int J Cell Biol 2014: 981750, 2014

13. Shen X, Wang S, Wang H, Liang M, Xiao L and Wang Z: The role of SDF-1/CXCR4 axis in ovarian cancer metastasis. J Huazhong Univ Sci Technolog Med Sci 29: 363-367, 2009.

14. Zhou W, Guo S, Liu M, Burow ME and Wang G: Targeting CXCL12/CXCR4 axis in tumor immunotherapy. Curr Med Chem 26: 3026-3041, 2019. 
15. Chen Y, Ramjiawan RR, Reiberger T, Ng MR, Hato T, Huang Y, Ochiai H, Kitahara S, Unan EC, Reddy TP, et al: CXCR4 inhibition in tumor microenvironment facilitates anti-programmed death receptor-1 immunotherapy in sorafenib-treated hepatocellular carcinoma in mice. Hepatology 61: 1591-1602, 2015.

16. Wald O: CXCR4 based therapeutics for non-small cell lung cancer (NSCLC). J Clin Med 7: 303, 2018.

17. Otsuka S and Bebb G: The CXCR4/SDF-1 chemokine receptor axis: A new target therapeutic for non-small cell lung cancer. J Thorac Oncol 3: 1379-1383, 2008.

18. Wang B, Wang W, Niu W, Liu E, Liu X, Wang J, Peng C, Liu S, $\mathrm{Xu}$ L, Wang L and Niu J: SDF-1/CXCR4 axis promotes directional migration of colorectal cancer cells through upregulation of integrin alphavbeta6. Carcinogenesis 35: 282-291, 2014.

19. Walentowicz-Sadlecka M, Sadlecki P, Bodnar M, Marszalek A, Walentowicz P, Sokup A, Wilińska-Jankowska A and Grabiec M Stromal derived factor- 1 (SDF-1) and its receptors CXCR4 and CXCR7 in endometrial cancer patients. PLoS One 9: e84629. 2014.

20. Mao W, Yi X, Qin J, Tian M and Jin G: CXCL12/CXCR4 axis improves migration of neuroblasts along corpus callosum by stimulating MMP-2 secretion after traumatic brain injury in rats. Neurochem Res 41: 1315-1322, 2016.

21. Ahirwar DK, Nasser MW, Ouseph MM, Elbaz M, Cuitiño MC, Kladney RD, Varikuti S, Kaul K, Satoskar AR, Ramaswamy B, et al: Fibroblast-derived CXCL12 promotes breast cancer metastasis by facilitating tumor cell intravasation. Oncogene 37: 4428-4442, 2018.

22. Lang J, Zhao X, Qi Y, Zhang Y, Han X, Ding Y, Guan J, Ji T, Zhao Y and Nie G: Reshaping prostate tumor microenvironment To suppress metastasis via cancer-associated fibroblast inactivation with peptide-assembly-based nanosystem. ACS Nano 13 12357-12371, 2019.

23. Mahadevan D and Von Hoff DD: Tumor-stroma interactions in pancreatic ductal adenocarcinoma. Mol Cancer Ther 6 : 1186-1197, 2007

24. Zeng Z, Shi YX, Samudio IJ, Wang RY, Ling X, Frolova O, Levis M, Rubin JB, Negrin RR, Estey EH, et al: Targeting the leukemia microenvironment by CXCR4 inhibition overcomes resistance to kinase inhibitors and chemotherapy in AML. Blood 113: 6215-6224, 2009.

25. Kong L, Guo S, Liu C, Zhao Y, Feng C, Liu Y, Wang T and Li C: Overexpression of SDF-1 activates the NF-kappaB pathway to induce epithelial to mesenchymal transition and cancer stem cell-like phenotypes of breast cancer cells. Int J Oncol 48 1085-1094, 2016

26. Kim SY, Lee CH, Midura BV, Yeung C, Mendoza A, Hong SH, Ren L, Wong D, Korz W, Merzouk A, et al: Inhibition of the CXCR4/CXCL12 chemokine pathway reduces the development of murine pulmonary metastases. Clin Exp Metastasis 25: 201-211, 2008

27. Meng W, Xue S and Chen Y: The role of CXCL12 in tumor microenvironment. Gene 641: 105-110, 2018.

28. Conley-LaComb MK, Semaan L, Singareddy R, Li Y, Heath EI, Kim S, Cher ML and Chinni SR: Pharmacological targeting of CXCL12/CXCR4 signaling in prostate cancer bone metastasis. Mol Cancer 15: 68, 2016

29. Ray P, Lewin SA, Mihalko LA, Schmidt BT, Luker KE and Luker GD: Noninvasive imaging reveals inhibition of ovarian cancer by targeting CXCL12-CXCR4. Neoplasia 13: 1152-1161, 2011.

30. Li H, Chen Y, Xu N, Yu M, Tu X, Chen Z, Lin M, Xie B, Fu J and Han L: AMD3100 inhibits brain-specific metastasis in lung cancer via suppressing the SDF-1/CXCR4 axis and protecting blood-brain barrier. Am J Transl Res 9: 5259-5274, 2017.

31. Tong X, Ma Y, Deng H, Wang X, Liu S, Yan Z, Peng S and Fan H: The SDF-1 rs1801157 polymorphism is associated with cancer risk: An update pooled analysis and FPRP test of 17,876 participants. Sci Rep 6: 27466, 2016.

32. Ponting CP: Novel repeats in ryanodine and IP 3 receptors and protein O-mannosyltransferases. Trends Biochem Sci 25: 48-50, 2000.

33. Hamada T, Tashiro K, Tada H, Inazawa J, Shirozu M, Shibahara K, Nakamura T, Martina N, Nakano T and Honjo T: Isolation and characterization of a novel secretory protein, stromal cell-derived factor-2 (SDF-2) using the signal sequence trap method. Gene 176: 211-214, 1996.

34. Vendrell E, Ribas M, Valls J, Solé X, Grau M, Moreno V, Capellà $\mathrm{G}$ and Peinado MA: Genomic and transcriptomic prognostic factors in R0 Dukes B and C colorectal cancer patients. Int J Oncol 30: 1099-1107, 2007
35. Fukuda S, Sumii M, Masuda Y, Takahashi M, Koike N, Teishima J, Yasumoto H, Itamoto T, Asahara T, Dohi K and Kamiya K: Murine and human SDF2L1 is an endoplasmic reticulum stress-inducible gene and encodes a new member of the Pmt/rt protein family. Biochem Biophys Res Commun 280: 407-414, 2001

36. Lorenzon-Ojea AR, Caldeira W, Ribeiro AF, Fisher SJ, Guzzo CR and Bevilacqua E: Stromal cell derived factor-2 (Sdf2): A novel protein expressed in mouse. Int J Biochem Cell Biol 53: 262-270, 2014.

37. Lorenzon-Ojea AR, Yung HW, Burton GJ and Bevilacqua E: The potential contribution of stromal cell-derived factor 2 (SDF2) in endoplasmic reticulum stress response in severe preeclampsia and labor-onset. Biochim Biophys Acta Mol Basis Dis 1866: 165386, 2020.

38. Walter P and Ron D: The unfolded protein response: From stress pathway to homeostatic regulation. Science 334: 1081-1086, 2011

39. Schott A, Ravaud S, Keller S, Radzimanowski J, Viotti C, Hillmer S, Sinning I and Strahl S: Arabidopsis stromal-derived Factor2 (SDF2) is a crucial target of the unfolded protein response in the endoplasmic reticulum. J Biol Chem 285: 18113-18121, 2010.

40. Willis S, Villalobos VM, Gevaert O, Abramovitz M, Williams C, Sikic BI and Leyland-Jones B: Single gene prognostic biomarkers in ovarian cancer: A meta-analysis. PLoS One 11: e0149183, 2016.

41. Giulianelli S, Herschkowitz JI, Patel V, Lamb CA, Gutkind JS, Molinolo A, Perou CM and Lanari C: MPA-induced gene expression and stromal and parenchymal gene expression profiles in luminal murine mammary carcinomas with different hormonal requirements. Breast Cancer Res Treat 129: 49-67, 2011.

42. Takahashi K, Tanaka M, Yashiro M, Matsumoto M, Ohtsuka A, Nakayama KI, Izumi Y, Nagayama K, Miura K, Iwao H and Shiota M: Protection of stromal cell-derived factor 2 by heat shock protein 72 prevents oxaliplatin-induced cell death in oxaliplatin-resistant human gastric cancer cells. Cancer Lett 378: $8-15,2016$

43. HonoréB: The rapidly expanding CREC protein family: Members, localization, function, and role in disease. Bioessays 31: 262-277, 2009.

44. Luo J, Li Z, Zhu H, Wang C, Zheng W, He Y, Song J, Wang W, Zhou X, Lu X, et al: A novel role of Cab45-G in mediating cell migration in cancer cells. Int J Biol Sci 12: 677-687, 2016.

45. Honoré B and Vorum H: The CREC family, a novel family of multiple EF-hand, low-affinity $\mathrm{Ca}(2+)$-binding proteins localised to the secretory pathway of mammalian cells. FEBS Lett 466 : $11-18,2000$

46. Scherer PE, Lederkremer GZ, Williams S, Fogliano M, Baldini G and Lodish HF: Cab45, a novel (Ca2+)-binding protein localized to the Golgi lumen. J Cell Biol 133: 257-268, 1996.

47. Chen L, Xu S, Xu Y, Lu W, Liu L, Yue D, Teng J and Chen J: Cab45S promotes cell proliferation through SERCA2b inhibition and $\mathrm{Ca} 2+$ signaling. Oncogene 35: 35-46, 2016.

48. Lam PP, Hyvärinen K, Kauppi M, Cosen-Binker L, Laitinen S, Keränen S, Gaisano HY and Olkkonen VM: A cytosolic splice variant of Cab45 interacts with Munc18b and impacts on amylase secretion by pancreatic acini. Mol Biol Cell 18: 2473-2480, 2007.

49. Grønborg M, Kristiansen TZ, Iwahori A, Chang R, Reddy R, Sato N, Molina H, Jensen ON, Hruban RH, Goggins MG, et al: Biomarker discovery from pancreatic cancer secretome using a differential proteomic approach. Mol Cell Proteomics 5: 157-171, 2006.

50. Ji H, Greening DW, Kapp EA, Moritz RL and Simpson RJ: Secretome-based proteomics reveals sulindac-modulated proteins released from colon cancer cells. Proteomics Clin Appl 3: 433-451, 2009.

51. Blank B and von Blume J: Cab45-Unraveling key features of a novel secretory cargo sorter at the trans-Golgi network. Eur J Cell Biol 96: 383-390, 2017.

52. Mittal V: Epithelial mesenchymal transition in tumor metastasis. Annu Rev Pathol 13: 395-412, 2018.

53. Gialeli C, Theocharis AD and Karamanos NK: Roles of matrix metalloproteinases in cancer progression and their pharmacological targeting. FEBS J 278: 16-27, 2011.

54. Bisel B, Wang Y, Wei JH, Xiang Y, Tang D, Miron-Mendoza M, Yoshimura S, Nakamura N and Seemann J: ERK regulates Golgi and centrosome orientation towards the leading edge through GRASP65. J Cell Biol 182: 837-843, 2008. 
55. Prigozhina NL and Waterman-Storer CM: Protein kinase D-mediated anterograde membrane trafficking is required for fibroblast motility. Curr Biol 14: 88-98, 2004.

56. Kienzle $\mathrm{C}$ and von Blume J: Secretory cargo sorting at the trans-Golgi network. Trends Cell Biol 24: 584-593, 2014.

57. Weiss H, Amberger A, Widschwendter M, Margreiter R, Ofner D and Dietl P: Inhibition of store-operated calcium entry contributes to the anti-proliferative effect of non-steroidal anti-inflammatory drugs in human colon cancer cells. Int J Cancer 92: 877-882, 2001.

58. Yilmaz $\mathrm{M}$ and Christofori G: EMT, the cytoskeleton, and cancer cell invasion. Cancer Metastasis Rev 28: 15-33, 2009.

59. Kessenbrock K, Plaks V and Werb Z: Matrix metalloproteinases: Regulators of the tumor microenvironment. Cell 141: 52-67, 2010.

60. Merchant N, Nagaraju GP, Rajitha B, Lammata S, Jella KK, Buchwald ZS, Lakka SS and Ali AN: Matrix metalloproteinases: Their functional role in lung cancer. Carcinogenesis 38: 766-780, 2017.

61. Shirozu M, Tada H, Tashiro K, Nakamura T, Lopez ND Nazarea M, Hamada T, Sato T, Nakano T and Honjo T: Characterization of novel secreted and membrane proteins isolated by the signal sequence trap method. Genomics 37 : 273-280, 1996

62. Shi Y, He B, You L and Jablons DM: Roles of secreted frizzled-related proteins in cancer. Acta Pharmacol Sin 28 : 1499-1504, 2007.

63. Liu Y, Zhou Q, Zhou D, Huang C, Meng X and Li J: Secreted frizzled-related protein 2-mediated cancer events: Friend or foe? Pharmacol Rep 69: 403-408, 2017.

64. Arce L, Yokoyama NN and Waterman ML: Diversity of LEF/TCF action in development and disease. Oncogene 25 7492-7504, 2006.

65. Zhang X, Rong X, Chen Y and Su L: Methylation-mediated loss of SFRP2 enhances invasiveness of non-small cell lung cancer cells. Hum Exp Toxicol 37: 155-162, 2018.

66. Li P, Zhao S and Hu Y: SFRP2 modulates nonsmall cell lung cancer A549 cell apoptosis and metastasis by regulating mitochondrial fission via Wnt pathways. Mol Med Rep 20: 1925-1932, 2019.

67. Zeng X, Zhang Y, Xu H, Zhang T, Xue Y and An R: Secreted frizzled related protein 2 modulates epithelial-mesenchymal transition and stemness via Wnt/ $\beta$-catenin signaling in choriocarcinoma. Cell Physiol Biochem 50: 1815-1831, 2018

68. Cheng YY, Yu J, Wong YP, Man EP, To KF, Jin VX, Li J, Tao Q Sung JJ, Chan FK and Leung WK: Frequent epigenetic inactivation of secreted frizzled-related protein 2(SFRP2) by promoter methylation in human gastric cancer. Br J Cancer 97: 895-901, 2007.

69. Perry AS, O'Hurley G, Raheem OA, Brennan K, Wong S, O'Grady A, Kennedy AM, Marignol L, Murphy TM, Sullivan L, et al: Gene expression and epigenetic discovery screen reveal methylation of SFRP2 in prostate cancer. Int J Cancer 132: 1771-1780, 2013.

70. Bhangu JS, Beer A, Mittlböck M, Tamandl D, Pulverer W, Schönthaler S, Taghizadeh H, Stremitzer S, Kaczirek K, Gruenberger $\mathrm{T}$, et al: Circulating free methylated tumor DNA markers for sensitive assessment of tumor burden and early response monitoring in patients receiving systemic chemotherapy for colorectal cancer liver metastasis. Ann Surg 268: 894-902, 2018
71. Luo X, Wei B, Chen A, Zhao H, Huang $\mathrm{K}$ and Chen J: Methylation-mediated loss of SFRP2 enhances melanoma cell invasion via Wnt signaling. Am J Transl Res 8: 1502-1509, 2016.

72. Liu S, Chen X, Chen R, Wang J, Zhu G, Jiang J, Wang H, Duan S and Huang J: Diagnostic role of Wnt pathway gene promoter methylation in non small cell lung cancer. Oncotarget 8: 36354-36367, 2017.

73. Shih YL, Hsieh CB, Yan MD, Tsao CM, Hsieh TY, Liu CH and Lin YW: Frequent concomitant epigenetic silencing of SOX1 and secreted frizzled-related proteins (SFRPs) in human hepatocellular carcinoma. J Gastroenterol Hepatol 28: 551-559, 2013.

74. Yang Q, Huang T, Ye G, Wang B and Zhang X: Methylation of SFRP2 gene as a promising noninvasive biomarker using feces in colorectal cancer diagnosis: A systematic meta-analysis. Sci Rep 6: 33339, 2016.

75. Lee JL, Lin CT, Chueh LL and Chang CJ: Autocrine/paracrine secreted Frizzled-related protein 2induces cellular resistance to apoptosis: A possible mechanism of mammary tumorigenesis. J Biol Chem 279: 14602-14609, 2004.

76. Courtwright A, Siamakpour-Reihani S, Arbiser JL, Banet N, Hilliard E, Fried L, Livasy C, Ketelsen D, Nepal DB, Perou CM, et al: Secreted frizzle-related protein 2 stimulates angiogenesis via a calcineurin/NFAT signaling pathway. Cancer Res 69: 4621-4628, 2009

77. Lee JL, Chang CJ, Chueh LL and Lin CT: Secreted frizzled related protein 2(sFRP2) decreases susceptibility to UV-induced apoptosis in primary culture of canine mammary gland tumors by NF-kappaB activation or JNK suppression. Breast Cancer Res Treat 100: 49-58, 2006.

78. Lee JL, Chang CJ, Wu SY, Sargan DR and Lin CT: Secreted frizzled-related protein 2(SFRP2) is highly expressed in canine mammary gland tumors but not in normal mammary glands. Breast Cancer Res Treat 84: 139-149, 2004.

79. Viros A, Girotti MR and Marais R: So you can teach old fibroblasts new tricks. Cancer Discov 6: 581-583, 2016.

80. Fontenot E, Rossi E, Mumper R, Snyder S, Siamakpour-Reihani S, Ma P, Hilliard E, Bone B, Ketelsen D, Santos C, et al: A novel monoclonal antibody to secreted frizzled-related protein 2 inhibits tumor growth. Mol Cancer Ther 12: 685-695, 2013.

81. Xiao X, Xiao Y, Wen R, Zhang Y, Li X, Wang H, Huang J, Liu J, Long T and Tang J: Promoting roles of the secreted frizzled-related protein 2as a Wnt agonist in lung cancer cells. Oncol Rep 34: 2259-2266, 2015.

82. Zhang Y, Li Q and Chen H: DNA methylation and histone modifications of Wnt genes by genistein during colon cancer development. Carcinogenesis 34: 1756-1763, 2013.

83. Wang WA, Groenendyk J and Michalak M: Endoplasmic reticulum stress associated responses in cancer. Biochim Biophys Acta 1843: 2143-2149, 2014

This work is licensed under a Creative Commons Attribution-NonCommercial-NoDerivatives 4.0 International (CC BY-NC-ND 4.0) License. 